\title{
Using hyperspectral remote sensing data in urban mapping over Kuala Lumpur
}

\begin{abstract}
Hyperspectral remote sensing has great application potential for analyzing complex urban scenes. In this study, airborne hyperspectral data over part of Kuala Lumpur, Malaysia were used to classify 14 urban classes. In order to do the classification, Support Vector Machine (SVM) was used. Some filters (Lee and Enhanced Lee) were used before performing the classification. Consequently, the results showed that the overall accuracy is improved (3\%$4 \%$ ) when the filters were applied to the image. The overall accuracy for classification of the study area using SVM is $89 \%$ with Kappa coefficient 0.88 without filtering. The use of Lee and Enhanced Lee filters improved the accuracy to 92 and $93.6 \%$ respectively. This study serves as a pioneering effort in the application of hyperspectral sensing for urban area in Malaysia.
\end{abstract}

Keyword: Hyperspectral remote sensing; Kuala Lumpur; Hyperspectral sensing; Urban mapping 